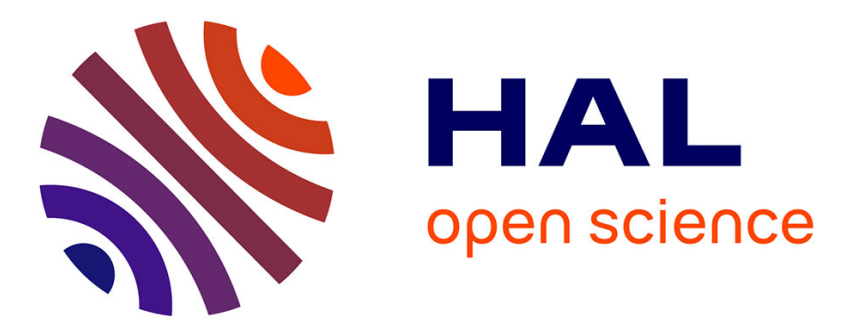

\title{
Towards the evidence of a purely spatial spatial Einstein-Podolsky-Rosen paradox in images: measurement scheme and first experimental results
}

Fabrice Devaux, Joé Mougin-Sisini, Paul-Antoine Moreau, Éric Lantz

\section{- To cite this version:}

Fabrice Devaux, Joé Mougin-Sisini, Paul-Antoine Moreau, Éric Lantz. Towards the evidence of a purely spatial spatial Einstein-Podolsky-Rosen paradox in images: measurement scheme and first experimental results. The European Physical Journal D: Atomic, molecular, optical and plasma physics, 2012, 66, pp.192. 10.1140/epjd/e2012-20695-2 . hal-00949059

\section{HAL Id: hal-00949059 \\ https://hal.science/hal-00949059}

Submitted on 19 Feb 2014

HAL is a multi-disciplinary open access archive for the deposit and dissemination of scientific research documents, whether they are published or not. The documents may come from teaching and research institutions in France or abroad, or from public or private research centers.
L'archive ouverte pluridisciplinaire HAL, est destinée au dépôt et à la diffusion de documents scientifiques de niveau recherche, publiés ou non, émanant des établissements d'enseignement et de recherche français ou étrangers, des laboratoires publics ou privés. 


\title{
Towards the evidence of a purely spatial Einstein-Podolsky-Rosen
}

\section{paradox in images: measurement scheme and first experimental}

\section{results}

Fabrice Devaux, Joé Mougin-Sisini, Paul-Antoine Moreau, and Eric Lantz

Département d'Optique P.M. Duffieux, Institut FEMTO-ST, UMR CNRS 6174, Université de Franche-Comté, 16 route de Gray, F-25030 Besançon Cedex, France

Received: date / Revised version: date

\begin{abstract}
We propose a scheme to evidence the Einstein-Podolsky-Rosen (EPR) paradox for photons produced by spontaneous down conversion, from measurement of purely spatial correlations of photon positions both in the near and in the far-field. Experimentally, quantum correlations have been measured in the far-field of parametric fluorescence created in a type II BBO crystal. Imaging is performed in the photon counting regime with an electron-multiplying CCD (EMCCD) camera.
\end{abstract}

PACS. 42.50.Ar Photon statistics and coherence theory - 42.50.Lc Quantum fluctuations, quantum noise and quantum jumps

\section{Introduction}

Spontaneous parametric down conversion (SPDC) of a wide monomode gaussian pump results in a strongly multimode beam: the extension of the down converted beam in the near field (image plane)is identical to that of the pump, in the limit of low gain and for a sufficiently wide crystal, while the far field (Fourier plane) extension is lim-

Send offprint requests to: Fabrice Devaux Correspondence to: fabrice.devaux@univ-fcomte.fr ited by phase-matching. The etendue of the beam, i.e. the product of its transverse surface by the solid angle it subtends or the number of transverse modes in appropriate units (resolution cells in ref. [1]), has been recognized [2, 3] as corresponding to the two-photon Schmidt number. Note that the spatial extension of a mode in either the near or the far-field is proportional to the inverse of the full beam extension in the other plane. For single photon imaging, the laws of diffraction are equivalent to the 
Heisenberg's uncertainty relation: a photon that can be localized in one mode of the near field, for example by traversing an aperture of the size corresponding to the mode, will be detected at a random position in the entire far-field diffraction pattern. However, the laws of quantum mechanics state that a pair of signal-idler photons will be detected either in the same mode in the near field or in opposite modes in the far field, if no detection occurs in the other plane. Because the detection plane can be chosen at a time where causal interaction between photons is no more possible, these correlations are not compatible with local realism, as demonstrated first in the famous EPR paper [4], though compatible with the Heisenberg's uncertainty relation since correlations cannot be measured in both planes for the same photon pair.

For a sufficiently low SPDC rate, it can be assumed that a pair does not interact with another, which makes the system very close of that considered in the original EPR paper: the positions of photons 1 and 2 are detected in the near-field and their momenta correspond to the farfield. Howell et al. [5] have measured in both planes the probability distribution of the position of photon 2 , conditioned by the detection of photon 1 . The product of the conditional variances is 25 times smaller than the limit for the product of variances for a single photon given by the Heisenberg's uncertainty relation. This impressive result has been obtained by measuring temporal coincidences between cross-polarized photons in type 2 SPDC. These photons were separated by a polarizing beam-splitter: for a fixed position of a narrow slit transmitting the photon 1 to an avalanche photodiode, the level of coincidences was measured for each position of a similar slit transmitting the photon 1 to a separate similar detector.

Three directions can be considered in order to add to the results of ref [5]. First, for a detection in this experiment of a photon 1 , the photon 2 is not detected but at a precise position, resulting in a vast majority of single detections without coincidences. In the words of Reid et al. [6] about the Aspect et al. [7] experiments: "However, it is debatable whether this can be regarded as a rigorous EPR experiment because for the full ensemble, most counts at one detector correspond to no detection at the other". The situation is comparable in the Howell et al experiment, though with other conjugate variables (position-momentum instead of polarizations). Note that the light in several transverse modes has been simultaneously recorded in a recent experiment [8].

Second, the asymmetry between photons 1 and 2 could be relaxed [6], even if this asymmetry is present in the original EPR paper. Third, the assumption of a pure biphoton state [9] does not correspond to a general model of parametric amplification of quantum noise: beyond the limit of a very low gain, stimulated pairs remain perfectly correlated [10]. We propose in this paper a purely spatial detection scheme that has some advantages as regards the two first points, while assuming strictly spontaneous conversion without further amplification like in ref. [5]. The discussion of this third point is left for the conclusion section.

In recent papers, we have experimentally demonstrated, 
using an EMCCD camera in the photon-counting regime, that far-field opposite spatial fluctuations of type 1 SPDC are correlated in the quantum regime, with a variance of the photon number difference between opposite areas below the shot-noise level. This conclusion holds close to degeneracy for opposite pixels [11], as well as for broad-band SPDC for opposite angular sectors [12]. To demonstrate EPR correlations, we must also show quantum correlations of signal-idler spatial fluctuations in the near field. Since an EMCCD cannot distinguish a pair from a single photon, a type 2 interaction in a $\mathrm{BBO}$ crystal is used, where the signal and idler photons can be separated because of their orthogonal polarizations. Hence, our experimental set-up is similar to that of Howell et al. until the polarization beam splitter, with a completely different detection scheme: images are formed by all detected photons for each polarization, without any temporal resolution, as in experiments of Jedrkiewicz at al. [13] and Brida et al [14] but in the photon-counting regime.

The paper is organized as follows. We propose in Section 2 a measurement procedure to evidence purely spatial EPR correlations. Section 3 is devoted to experimental results, in the far-field because presently the results in the nearfield are not still convincing. We quantify in section 4 the degree of apparent violation of the Heisenberg uncertainties that could be reached. Last, we discuss in the conclusion ways of improvement and extension to the stimulated regime.

\section{Purely spatial EPR correlations}

\subsection{Spatial measurements of EPR correlations}

For a detection of a photon 1 at $\overrightarrow{r_{1}}$, the probability density of detection of a photon 2 at $\overrightarrow{r_{2}}$ can be written as:

$$
p\left(\overrightarrow{r_{2}} \mid \overrightarrow{r_{1}}\right)=p\left(\overrightarrow{r_{2}}\right)+f(\Delta r)
$$

where $\mathrm{p}\left(\overrightarrow{r_{2}}\right)$ is the probability density of detection of a photon of another pair (accidental coincidences) and $f(\Delta r)$ is the probability density of detection of the twin photon, with $\Delta r=\left\|\overrightarrow{r_{2}} \pm \overrightarrow{r_{1}}\right\|$, + holding for the far-field (correlation of momenta on opposite modes) and - for the near-field. It is assumed translational invariance (this hypothesis will be further discussed in the next subsection), circular symmetry and independence of the pairs (pure SPDC without further amplification). Hence, if $\mathrm{N}_{1}$ is the number of photons 1 detected on a surface $\mathrm{S}_{1}$ and $\mathrm{N}_{2}$ the corresponding quantity for photons 2 , we have:

$$
\begin{aligned}
<N_{1} N_{2}> & =\int_{S_{1}} d r_{1}^{2} \int_{S_{2}} d r_{2}^{2} p\left(\overrightarrow{r_{1}} \text { and } \overrightarrow{r_{2}}\right) \\
& =\int_{S_{1}} d r_{1}^{2} \int_{S_{2}} d r_{2}^{2}\left\{p\left(\overrightarrow{r_{1}}\right) p\left(\overrightarrow{r_{2}}\right)+p\left(\overrightarrow{r_{1}}\right) f(\Delta r)\right\}
\end{aligned}
$$

Therefore, the probability of detection in $\mathrm{S}_{2}$ of the twin photon 2 of the photon 1 detected on $S_{1}$ is simply given by:

$$
F\left(S_{2}\right)=\int_{S_{2}} d r_{2}^{2} f(\Delta r)=\frac{<N_{1} N_{2}>-<N_{1}><N_{2}>}{<N_{1}>}
$$

If $S_{1}$ and $S_{2}$ have the same size, this expression can be symmetrized and becomes the normalized intercorrelation function:

$$
F\left(S_{2}\right)=F\left(S_{1}\right)=\frac{<N_{1} N_{2}>-<N_{1}><N_{2}>}{\left(<N_{1}>+<N_{2}>\right) / 2}
$$


For independent pairs, this quantity can also be expressed as a function of the variance of the difference between $\mathrm{N}_{1}$ and $\mathrm{N}_{2}$ :

$$
\begin{array}{r}
<N_{1}>=<N_{2}>=<\left(N_{1}\right)^{2}>-<N_{1}>^{2} \Rightarrow \\
F\left(S_{2}\right)=1-\frac{<\left(N_{1}-N_{2}\right)^{2}>}{<\left(N_{1}+N_{2}\right)>}
\end{array}
$$

Because of the translational invariance, the means in eq. 4 and 5 can be estimated by spatial averages on the different pixels in one image.

To summarize, we have linked the intercorrellation function with the conditional probabilities of detecting the twin SPDC photons. The width of the experimental intercorrelation will then be used to demonstrate an EPR violation of the Heisenberg inequalities. More details on the violation we expect to obtain are given in section 4 .

\section{Measurements of far-field spatial quantum correlations}

The experimental setup is sketched in Fig. 1a. The pump pulse at $355 \mathrm{~nm}$ provided by a passively Q-switch Nd:YAG laser (mean power: $27 \mathrm{~mW}$, pulse duration: $300 \mathrm{ps,} \mathrm{repe-}$ tition rate: $1 \mathrm{kHz}$ ), illuminates a $1 \mathrm{~mm}$ long type $2 \mathrm{BBO}$ nonlinear crystal. The far-field image of the parametric fluorescence is formed in the focal plane of a lens by a back-illuminated EMCCD camera from Andor Technology (Model iXon+ DU897-ECS-BV) with a quantum efficiency greater than $90 \%$ in the visible range. The detector area is formed by $512 \times 512$ pixels, with a pixel size of $16 \times 16 \mu \mathrm{m}^{2}$, or $0.46 \times 0.46 \mathrm{mrad}^{2}$ after division by the focal length. We used a readout rate of $10 \mathrm{MHz}$ at 14 bits and the camera was cooled to $-85^{\circ} \mathrm{C}$. Measurements at degeneracy were performed for a crystal orientation corresponding to collinear phase matching by using a narrowband interferential filter centered at $710 \mathrm{~nm}(\Delta \lambda=4 \mathrm{~nm})$. Photon counting regime was ensured by adjusting the exposure time such that the mean fluence of SPDC was between 0.1 and 0.2 photon per pixel [15].

With a pump beam diameter $\sim 2 \mathrm{~mm}$, the coherence area in the far-field is smaller than the area of a pixel. Moreover, the use of pump pulses with 300 ps duration (much longer than the coherence time of SPDC) and an exposure time of the EMCCD of 5 ms (i.e. 5 laser shots) allow the excess noise to be limited by increasing the number of temporal modes [16]: the mean number of photons for one spatiotemporal mode is less than $10^{-3}$, in good agreement with the hypothesis of pure spontaneous down conversion, without any stimulated amplification. Fig. 1b shows a sum of 50 images recorded with the EMCCD in the SPDC farfield. A type 2 far-field pattern is observed and the phase matching angular range $\left(\sim 65 \mathrm{~mm}^{-1} \mathrm{FWHM}\right)$ corresponds to 100 pixels. Fig. 2a shows the average of the intercorrelation between the signal and idler patterns (equivalent to eq. 4 ), where the surfaces $S_{1}$ and $S_{2}$ correspond to the physical pixels of the EMCCD. We have verified that no correlation exists between the signal and idler patterns for different images (fig. 2b). The total correlation can be estimated either by summing the correlation values between several physical pixels around those corresponding to the peak or by binning (grouping) the pixels before the calculation of the intercorrelation coefficients. A third so- 
(a)

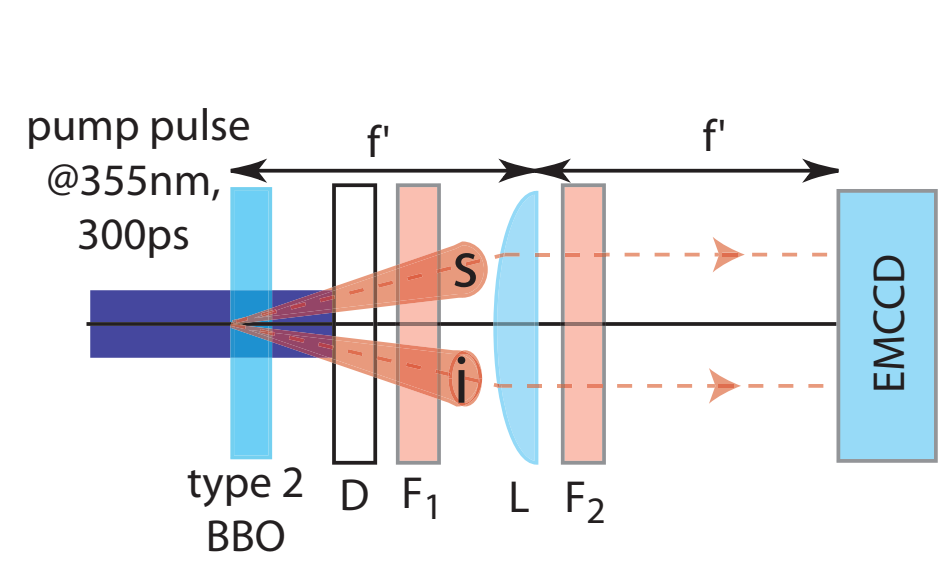

(b)

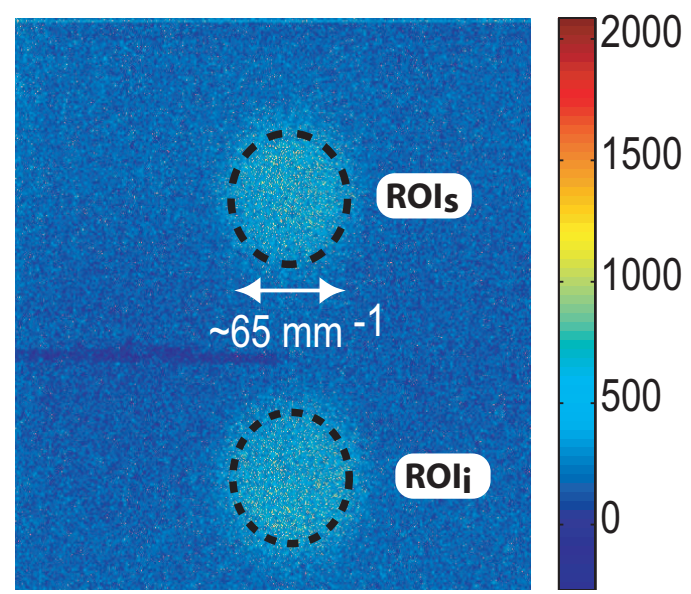

Fig. 1. (a): Far field experimental set-up. D : dichroic mirror, L: lens with focal length f'=35 mm, $F_{1}$ : broad-band red filter, $F_{2}$ : narrow-band interferential filter .(b): Sum of 50 far-field images of SPDC. The dotted circles denote signal and idler regions of interest (ROI) where statistics are performed.

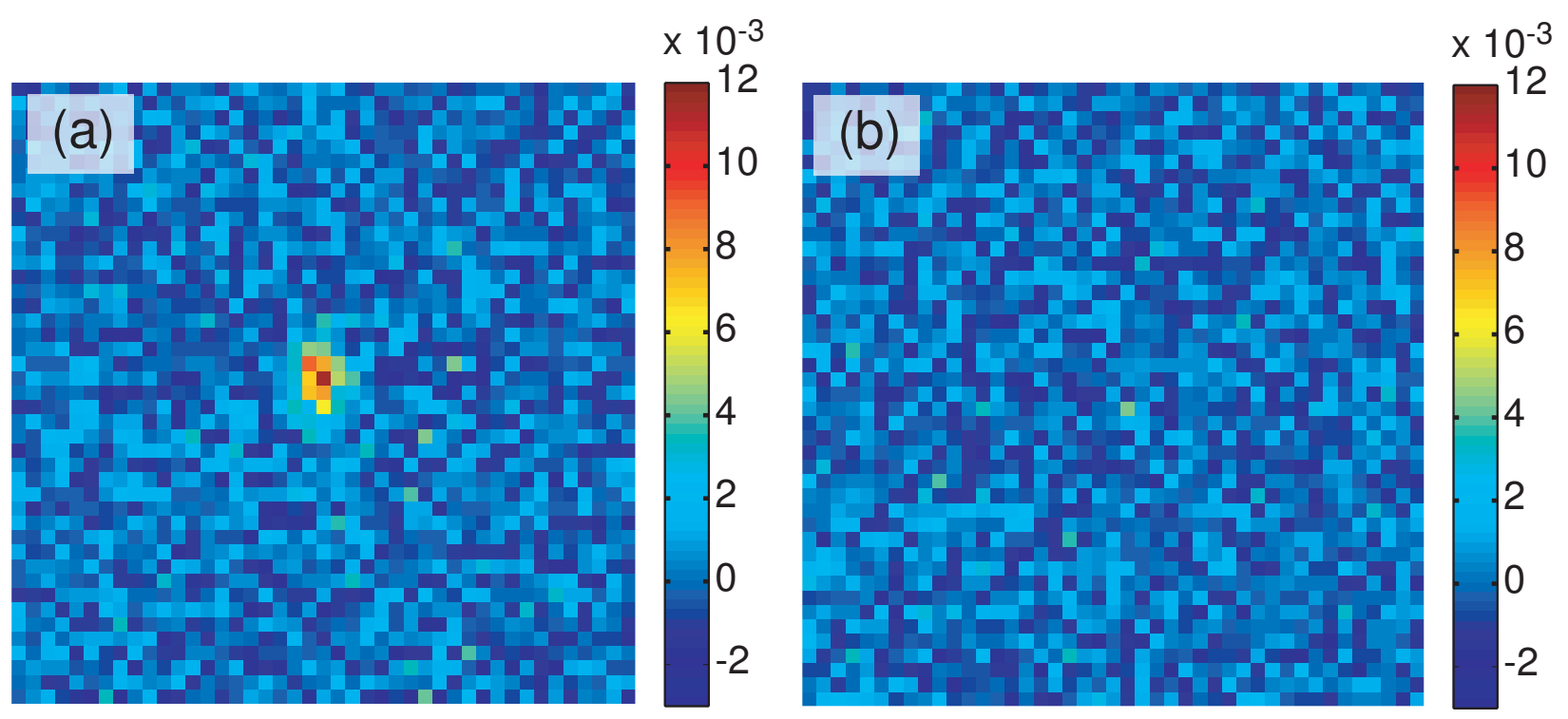

Fig. 2. (a):Average intercorrelation between signal and idler patterns.(b): Average intercorrelation between signal and idler patterns that come from different images.

lution consists in calculating the variance of the difference of the binned pixels (Eq. 5). Fig. 3 shows a comparison between these three methods. In all methods, a correction has been applied to take into account the small proba- bility of multiple photons impinging on the same pixel : see below. The agreement is good and the total intercorrelation coefficient of 0.1 is significant: all single detected photons contribute to a deterioration of this coefficient, 


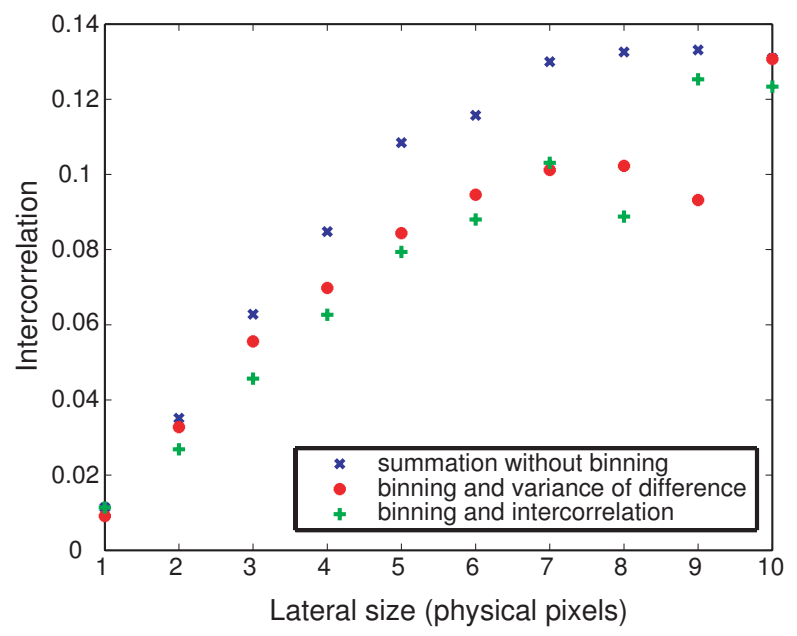

Fig. 3. Intercorrelation with respect to the pixel size (square pixels)

in contrast with experiments where only coincidences are considered. These single photons are due either to loss of the twin photon or to false positives caused by Clock Induced Noise [15]. We have also verified that the variance of the difference between opposite pixels is smaller than the shot noise level, as in our preceding papers in type 1 and in ref. [13] and [14] for type 2 in the high flux regime. Statistics are performed, for each individual image, on binned pixels inside the regions of interest (ROI) by measuring the difference between individual pixels in $\mathrm{ROI}_{s}$ and the symmetric pixels in $\mathrm{ROI}_{i}$ (fig. 1b). The variance of the difference can be expressed in shot noise units as: $r=c \frac{\sigma_{s-i}^{2}}{\left(m_{s}+m_{i}\right)} \cdot \sigma_{s-i}^{2}$ is the variance of the difference between symmetric pixels of $R O I_{s}$ and $R O I_{i}$, and $m_{s, i}$ is the mean, calculated respectively in $R O I_{s}$ and $R O I_{i}$. The correction coefficient $c=1 /\left(1-n_{\text {moy }}\right)$, where $n_{\text {moy }}$ is the measured mean on a physical pixel without binning, takes into account the fact that two photons or more can

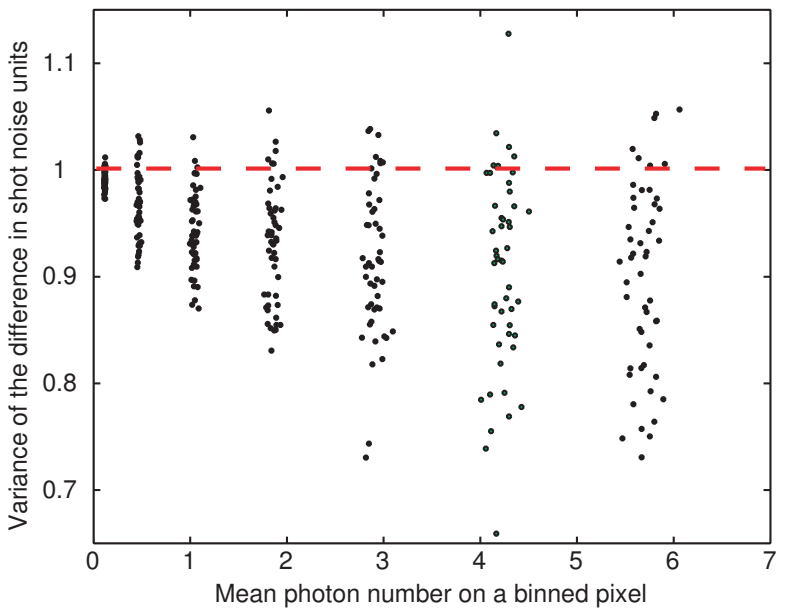

Fig. 4. Far-field variance of difference for individual images with respect to the mean number of photons in binned pixels, for binnings from $1 \times 1$ to $7 \times 7$.

be detected on the same physical pixel [11]. After this correction, the experimental spatial fluctuations on one signal or idler image obey a Poisson statistics: $c \frac{\sigma_{s}^{2}}{m_{s}}$ and $c \frac{\sigma_{i}^{2}}{m_{i}}$ are close to 1 . It can be easily shown that the same correction must be applied to the correlation coefficient (eq.4).

$r$ is calculated for each image and for different binnings. Fig. 4 shows the values of $r$ measured on 50 images: most of the measurements for individual images are below the shot noise level and the average of $r$ lies clearly in the quantum regime. For example, for a $5 \times 5$ binning, $\langle r\rangle=$ $0.916 \pm 0.020$, at $95 \%$ of confidence. Note that $r$ decreases and spreads out when increasing binning. The second feature comes from the smaller number of available pixels for the statistics.

Our experimental results in the near-field are not still convincing and are therefore not reported here. The most evident supplementary difficulty is the adding of a polarizer beam-splitter that induces losses and distortions. Note 
that these distortions must be eliminated over all the image field, unlike in the experiment of Howell et al where only a small part of the image plane is used [5]. Moreover, it has been shown [17] that the walk-off should be taken into account and depends on the crystal thickness. We plan to use a thinner crystal and to correct some geometrical aberrations in order to obtain convincing results in the near-field, that would allow the demonstration of a purely spatial EPR effect.

\section{Expected widths of EPR correlations}

In an ideal experiment, $\mathrm{F}$ tends to 1 if $\mathrm{S}_{1}$ (and $\mathrm{S}_{2}$ ) is greater than the coherence cell, in agreement with results of ref. [10]. We determine in this section the expected size of this coherence cell, in the far field as well as in the nearfield, and deduce the degree of violation of the Heisenberg criterion that can be attained by using conditional means.

We compare also the experimental results in the far-field with this expected size.

\subsection{Far field}

We first consider an experiment where an ideal narrow band interferential filter ensures a perfect frequency degeneracy: $\nu_{s}=\nu_{i}=\nu_{p} / 2$, where $\nu$ is the frequency of respectively the signal $\left(\nu_{s}\right)$, the idler $\left(\nu_{i}\right)$ and the pump $\left(\nu_{p}\right)$. The biphoton function reads in the far-field [18]:

$$
\widetilde{\psi}\left(q_{s}, q_{i}\right)=\chi \widetilde{E_{p}}\left(q_{s}+q_{i}\right) \widetilde{\zeta}\left(q_{s}, q_{i}\right)
$$

where $\chi$ is the coefficient of nonlinear interaction, $q_{s}$ and $q_{i}$ are the transverse spatial frequencies for the signal and the idler respectively, $\widetilde{E_{p}}$ is the pump field in the far-field and $\widetilde{\zeta}$ is the phase matching function. Eq. 6 states that the conditional probability function in the far-field is proportional to the pump amplitude in this plane, and therefore has the same width inasmuch as this width is much smaller than the width of the phase matching function. This condition is fully fulfilled in our experimental conditions: the phase matching function width is around $65 \mathrm{~mm}^{-1}$ ( see figure 1b), while the width of the pump field in the Fourier plane is around $0.5 \mathrm{~mm}^{-1}$ (see below), i.e. of the order of one pixel.

The situation is more complex if we take into account the non negligible width of the chromatic filter. Eq. 6 is still valid but the coordinates $f^{\prime} \sin \theta$ in the far-field detection plane are no more proportional to the transverse spatial frequencies. We have now $\sin \theta_{s}=\frac{q_{s} c / \nu_{s}}{2 \pi}, \sin \theta_{i}=\frac{q_{i} c / \nu_{i}}{2 \pi}$. This effect can be roughly quantified as follows. For a plane wave pump ensuring $q_{s}+q_{i}=0$, the shift $\Delta \theta$ of the idler photon position with respect to the position symmetrical of the signal photon is given by:

$$
\Delta \theta=\theta_{s}+\theta_{i}=\frac{q_{s} c}{2 \pi}\left(1 / \nu_{s}-1 / \nu_{i}\right)
$$

In a type 2 crystal, the centers of the SPDC patterns of the idler and the signal are separated in a noncritical configuration by the walk-off angle [19], i.e $120 \mathrm{mrad}$ at 710 nm: see fig.1b. The transmission of the interferential filter is gaussian with a standard deviation of $4 \mathrm{~nm}$. To obtain a rough estimate of the maximum value of $\Delta \theta$, we consider an equivalent rectangular filter with unity transmission, with the same total transmission after integration. The total width of this rectangular filter is $4 \sqrt{2 \pi}=10 \mathrm{~nm}$, 
giving for a couple of signal-idler photons at the edges of this filter $\Delta \theta=120 \times \frac{10}{710}=1.70 \mathrm{mrad}$, i.e 4 pixels. Because of the absence of walk-off, the effect is much weaker in the horizontal direction (angle $\varphi$ ), explaining the asymmetry in fig.2a.

To take into account more realistically the gaussian character of the filter and the phase matching function, the intercorrelation function in the far-field can be written as:

$$
\begin{gathered}
<N_{s} N_{i}>=\chi^{2} \int T\left(\nu_{s}\right) T\left(\nu_{p}-\nu_{s}\right) d \nu_{s} \int d \varphi_{p} \times \\
\int I_{p}\left(\varphi_{p}, \theta_{p}\right) d \theta_{p} \int d \varphi_{s} \int d \theta_{s}\left|\widetilde{\zeta}\left(\theta_{p}, \nu_{s}, \varphi_{s}-\varphi_{p}, \theta_{s}\right)\right|^{2}
\end{gathered}
$$

Where $\mathrm{T}$ is the transmission coefficient of the filter and $I_{p}$ is the pump intensity in the far-field. We have numerically calculated this intercorrelation function and determined its width by fitting it with a two-dimensional gaussian function. As expected, its shape is asymmetrical, with a standard deviation of 0.57 pixel in the horizontal direction and of 0.91 pixel in the vertical direction, for a theoretical value of 0.13 pixel at perfect degeneracy. In our experimental conditions, one pixel corresponds to $4.04 \hbar / \mathrm{mm}$ in momentum units. The same procedure applied to the experimental intercorrelation of fig.2 gives an horizontal standard deviation of 1.12 pixel and a vertical one of 1.74 pixel. It seems that some geometric aberrations in the optical system enlarge the correlation peak. Nevertheless, the asymmetry due to the non perfect degeneracy in temporal frequencies is visible with similar characteristics in simulation and experiment. Moreover, it can be shown that the mean probability of transmission by the filter of the twin photon is 0.56 . This relatively low number is one of the effect leading to an experimental total intercorrelation coefficient of 0.1 . The other important causes of reduction of this coefficient are the global quantum efficiency of the optical system, including all optics and the camera, the false positive or negative detections due to the camera [15], and probably also some residual fluorescence of the optical components.

\subsection{Near field}

We suppose first a perfect degeneracy of temporal frequencies. In the output plane of the crystal (we discuss at the end of this subsection how to take into account the imaging system), the biphoton function reads:

$$
\psi\left(x_{s}, x_{i}\right)=\int \chi E_{p}(x) \zeta\left(x_{s}+x, x_{i}+x\right) d x
$$

Let us first assume that the pump beam has a constant amplitude. In this case, the phase matching function in the direct space depends only of the difference of the coordinates [18]:

$$
\int \zeta\left(x_{s}+x, x_{i}+x\right) d x \equiv \gamma\left(x_{s}-x_{i}\right)
$$

where $\gamma(x)$ is the inverse Fourier transform of $\widetilde{\zeta}\left(q_{s},-q_{s}\right)$. We now justify our hypothesis: the width of $\widetilde{\zeta}$ is $65 \mathrm{~mm}^{-1}$, leading to a non negligible signal-idler correlation only for distances not much greater than $1 / 65 \mathrm{~mm}$. On this scale, we can safely assume that the $2 \mathrm{~mm}$ wide pump beam has a constant amplitude.

We have to find the standard deviation of $|\gamma(x)|^{2}$, proportional to the conditional probability $P\left(x_{s} \mid x_{i}\right)$. We have first:

$$
\widetilde{\zeta}\left(q_{s},-q_{s}\right)=\frac{\exp \left(i \Delta k_{z} L\right)-1}{i \Delta k_{z}}
$$


where $\Delta k_{z}$ is the longitudinal wave vector mismatch and $L$ the crystal length. By neglecting the index variation of the extraordinary wave versus the internal angle, $\Delta k_{z}$ becomes a purely geometrical term that reads, for perfect collinear phase matching:

$$
\Delta k_{z}=\frac{q_{s}^{2} \lambda}{4 \pi}\left(\frac{1}{n_{s}}+\frac{1}{n_{i}}\right)
$$

with $n_{s, i}$ the indices of the signal and the idler. The first zero of $\widetilde{\zeta}\left(q_{s},-q_{s}\right)$ is obtained for $q_{s 0}=2 \pi\left(\lambda L\left(\frac{1}{n_{s}}+\frac{1}{n_{i}}\right)\right)^{-1 / 2}$ We can now determine numerically the standard deviation $\sigma_{q_{s}}$ of the SPDC in the far-field:

$$
\sigma_{q_{s}}=\left(\int\left|\widetilde{\zeta}\left(q_{s},-q_{s}\right)\right|^{2} q_{s}^{2} d q_{s}\right)^{1 / 2}=0.69 q_{s 0}
$$

and the standard deviation $\sigma_{x_{i} \mid x_{s}}$ of the conditional probability in the direct space:

$$
\sigma_{x_{i} \mid x_{s}}=\left(\int|\gamma(x)|^{2} x^{2} d x\right)^{1 / 2}
$$

We find $\sigma_{x_{i} \mid x_{s}}=1.89 \sigma_{q_{s}}^{-1}$. Note that the same relation (with 1.88 instead of 1.89) has been given in [5], with no detail on the exact computation process.

Unlike in the far-field, a non perfect degeneracy does not modify these results: even if not of the same color, the twin photons are created at the same place, with an uncertainty proportional to the inverse of their spatial frequency bandwidth.

The effect of the imaging system is much more subtle. It has been analyzed in detail in [17]. A brief summary is as follows. Because the signal and idler wave vectors have directions shifted from the walk-off, the twin photons travel in the same direction in the crystal (the Poynting vectors are identical, see also [19]). However, a direction shift does exist in the imaging system and the images are laterally shifted. Moreover, to conserve a minimum $\sigma_{x_{i} \mid x_{s}}$, the transverse plane at the middle of the crystal (at equal distance between the input and the output face) must be imaged on the camera. With these precautions, the resolution found in eq. 14 is unaffected.

\subsection{Heisenberg violation using conditional probabilities}

The standard deviations in intensity of gaussian beams in the direct space $\sigma_{x}$ and in the Fourier space $\sigma_{q}$ obey the Heisenberg relation $\sigma_{x} \sigma_{q}=1 / 2$. In other words, if we admit that the intensity in a gaussian beam is proportional to the probability of presence of a photon, the Heisenberg uncertainty relation becomes equivalent to the standard diffraction theory. With the results of the preceding subsections, the ratio $\mathrm{R}$ of the Heisenberg variance product to the EPR variance product can be expressed as :

$$
R=\frac{\left(\sigma_{x} \sigma_{q}\right)^{2}}{\left(\sigma_{x_{i} \mid x_{s}} \sigma_{q_{i} \mid q_{s}}\right)^{2}}=\left(\frac{2 \pi 0.69}{1.89}\right)^{2} \frac{\sigma_{x}^{2}}{\lambda L\left(\frac{1}{n_{s}}+\frac{1}{n_{i}}\right)}
$$

In our experimental conditions : $\sigma_{x}=1 \mathrm{~mm}, L=1 \mathrm{~mm}$ and $\lambda=710 \mathrm{~nm}$ give $\sigma_{x_{i} \mid x_{s}}=0.013 \mathrm{~mm}, \hbar \sigma_{q_{i} \mid q_{s}}=0.52 \hbar / \mathrm{mm}$ and $R=5940$. If we admit that the interferential filter enlarges the correlation function in the far-field by a factor of 5 (see above), $R \approx 200$. Of course, other factors will probably diminish the experimental value by enlarging the correlation in the near field, like distortions due to the polarizing beam-splitter, other geometrical distortions, not perfect imaging of the middle plane of the crystal, etc... 
Nevertheless, eq.15 gives the conditions that allow a large violation of the Heisenberg limit, a thin crystal allowing a large phase-matching range and a wide pump beam. Once more, we stress that $R^{1 / 2}$ can also be interpreted, at perfect degeneracy, as the number of resolution cells in one transverse direction [1], or as the number of Schmidt modes [3].

\section{Conclusion}

We have proposed a scheme to demonstrate experimentally a purely spatial EPR paradox. Experimental results in the far-field are compatible with this objective and we have good hope to obtain soon the lacking results in the near-field. However, these results cannot easily be extended to higher fluxes because they are established with the assumption of independent pairs, or in more technical terms of a pure biphoton state. This limitation seems curious since even at high flux the signal-idler spatial fluctuations remain perfectly correlated, as demonstrated in numerous papers (see for example $[17,20]$ ). On the other hand, the demonstration of the EPR paradox for spatial properties of beams is based on a criterion implying the amplitude and phase quadratures [21,22]. While these quadrature operators are conjugate, with a $i \hbar$ commutator, the field operators respectively in the image plane and in the Fourier plane are Fourier transform each from the other and an EPR criterion in the continuous variable regime seems more difficult to establish. Of course, this curious asymmetry between the spatial and the temporal variables does not preclude the demonstration of purely spatial EPR, because experimentally the recorded intensities do correspond to purely spontaneous down conversion with totally negligible stimulated conversion.

\section{References}

1. F. Devaux and E. Lantz, Optics Communications 114 (1995) 114.

2. C. Law and J. Eberly, Phys. Rev. Lett. 12 (2004) 127903.

3. M. Van Exter, A. Aiello, S. Oemrawsingh, G. Nienhuis, and J. P. Woerdman, Phys. Rev. A 74 (2006) 012309.

4. A. Einstein, B. Podolsky, and N. Rosen, Phys. Rev. 47 (1935) 777 .

5. J. C. Howell, R.S. Bennink, S.J. Bentley, and R.W. Boyd, Phys. Rev. Lett. 92 (2004) 210403.

6. M. Reid, P. Drummond, W. Bowen, E. Cavalcanti, P. Lam, H. Bachor, U. Andersen, and G. Leuchs, Reviews of Modern Physics 81 (2009) 1727.

7. A. Aspect, P. Grangier, and G. Roger, Phys. Rev. Lett. 49 (1982) 91.

8. J.Leach, R.E.Warburton, D.G.Ireland, F.Izdebski, S.M.Barnett, A.M.Yao, G.S.Buller, and M.J.Padgett, Phys. Rev. A 85,(2012)013827

9. C. Monken, P. S. Ribeiro, and S. Padua, Phys. Rev. A 57 (1998) 3123.

10. A. Gatti, E. Brambilla, and L. A. Lugiato, Phys. Rev. Lett. 90 (2003) 133603.

11. J.L. Blanchet, F. Devaux, L. Furfaro, and E. Lantz, Phys. Rev. A 81 (2010) 043825.

12. J.L. Blanchet, F. Devaux, L. Furfaro, and E. Lantz, Phys. Rev. Lett. 101 (2008) 233604. 
13. O. Jedrkiewicz, Y.K. Jiang, E. Brambilla, A. Gatti, M. Bache, L. A. Lugiato, and P. Di Trapani, Phys. Rev. Lett. 93 (2004) 243601.

14. G. Brida, M. Genovese, and I. R. Berchera, Nature Photonics 4 (2010) 227.

15. E. Lantz, J.L. Blanchet, L. Furfaro, and F. Devaux, MNRAS 386 (2008) 2262.

16. E. Brambilla, L. Caspani, O. Jedrkiewicz, L.A. Lugiato, and A. Gatti, Phys. Rev. A 77 (2008) 053807.

17. E. Brambilla, A. Gatti, M. Bache, and L.A. Lugiato, Phys. Rev. A 69 (2004) 023802.

18. B.E.A. Saleh, A.F. Abouraddy, A. V. Sergienko, and M. C. Teich, Phys. Rev. A 62 (2000) 043816.

19. E. Lantz , F. Devaux, Journal of Optics A 2 (2000), 362364

20. E. Lantz, N. Treps, C. Fabre, and E. Brambilla, European Physical Journal D 29 (2004) 437.

21. K. Wagner, J. Janousek, V. Delaubert, H. Zou, C. Harb, N. Treps, J.F. Morizur, P.K. Lam, and H.A. Bachor, Science 321 (2008) 541.

22. V. Boyer, A. M. Marino, R. C. Pooser, and P.D. Lett, Science 321 (2008) 544 . 